\title{
Deep Radio Imaging with MERLIN of the Supernova Remnants in M82
}

\author{
T.W.B. Muxlow ${ }^{1}$, A. Pedlar ${ }^{1}$, J.D. Riley ${ }^{1}$, A.R. McDonald ${ }^{1}$, R.J. Beswick ${ }^{1}$, \\ and K.A. Wills ${ }^{2}$ \\ 1 Jodrell Bank Observatory, University of Manchester, Macclesfield, Cheshire \\ SK11 9DL; UK; \\ twbm@jb.man.ac.uk \\ 2 Department of Physics and Astronomy, University of Sheffield, Sheffield S3 \\ 7RH. UK
}

Summary. An 8 day MERLIN deep integration at $5 \mathrm{GHz}$ of the central region of the starburst galaxy M82 has been used to investigate the radio structure of a number of supernova remnants in unprecedented detail revealing new shells and partial shell structures for the first time. In addition, by comparing the new deep 2002 image with an astrometrically aligned image from 36 hours of data taken in 1992, it has been possible to directly measure the expansion velocities of 4 of the most compact remnants in M82. For the two most compact remnants, $41.95+575$ and $43.31+592$, expansion velocities of $2800 \pm 300 \mathrm{~km} \mathrm{~s}^{-1}$ and $8750 \pm 400 \mathrm{~km} \mathrm{~s}^{-1}$ have been derived. These confirm and refine the measured expansion velocities which have been derived from VLBI multi-epoch studies. For remnants $43.18+583$ and $44.01+596$, expansion velocities of $10500 \pm 750 \mathrm{~km} \mathrm{~s}^{-1}$ and $2400 \pm 250 \mathrm{~km} \mathrm{~s}^{-1}$ have been measured for the first time. In addition, the peak of the radio emission for SNR $45.17+612$ has moved between the two epochs implying velocities around $7500 \mathrm{~km} \mathrm{~s}^{-1}$. The relatively compact remnants in M82 are thus found to be expanding over a wide range of velocities which appear unrelated to their size. The new 2002 map is the most sensitive high-resolution image yet made of M82, achieving an $\mathrm{rms}$ noise level of $17 \mu \mathrm{Jy}_{\text {beam }}{ }^{-1}$. This establishes a first epoch for subsequent deep studies of expansion velocities for many SNR within M82.

\section{Introduction}

M82 is a well known nearby $(3.2 \mathrm{Mpc})$ starburst galaxy. The central $1 \mathrm{kpc}$ region contains at least 30 compact supernova remnants (SNR) with ages less than around 1000 years which have been imaged at high angular resolution by MERLIN [3] for over a decade. The most compact SNR have also been studied at very high angular resolution with VLBI $[2,4,5]$. All the SNR are resolved by MERLIN; however detailed expansion studies have been limited by the relatively poor signal-to-noise ratios of the earlier epoch images of many of the more resolved SNR. The 2002 epoch deep 8 day integration establishes the first MERLIN $5 \mathrm{GHz}$ image with high signal-to-noise ratios on the majority of well-resolved SNRs seen in M82. Subsequent deep images planned for later epochs will utilize the resurfaced Lovell antenna and new 


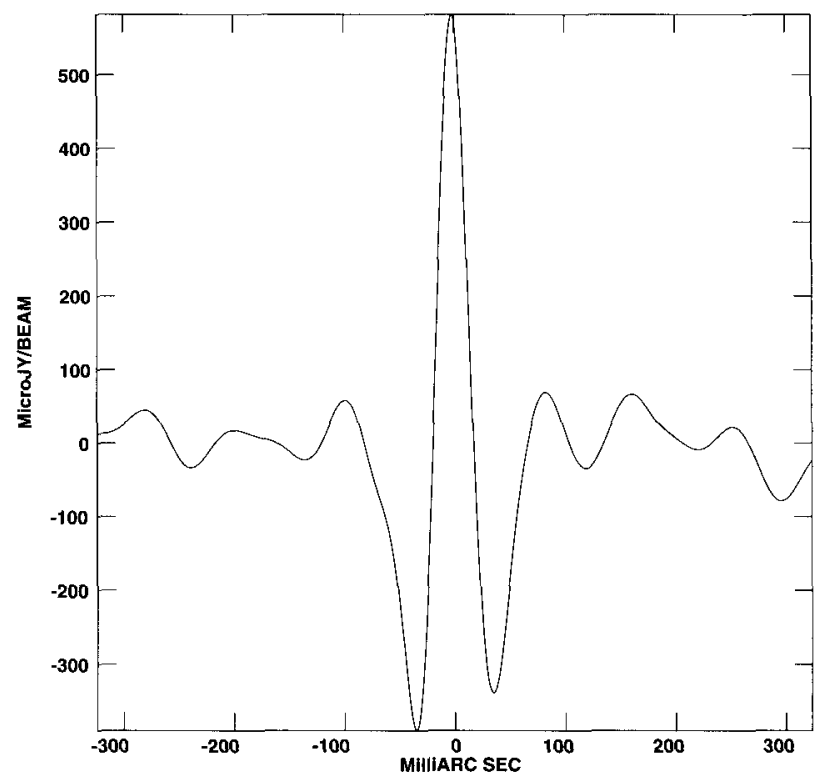

Fig. 1. Profile through the residual image of SNR $43.31+592$ after subtraction of the 2002 image from the 1992 map. The positive central and negative outer regions are indicative of expansion between the epochs.

more sensitive $5 \mathrm{GHz}$ receiver systems and optical-fiber wide-band links which are being developed for e-MERLIN. This will allow the detailed study of the evolution of the expanding shells to be made for the majority of SNR in M82.

\section{The MERLIN Observations}

Observations of M82 and the phase calibration source $0955+697$ lasting around 36 hours were made on $2-3$ July 1992 at $4994 \mathrm{GHz}$ with the 6 element MERLIN array. The deep 8 day MERLIN integration was observed between the 1 and 28 April 2002 at the same frequency. The flux density scale was calibrated from observations of the point source OQ208 and 3C286.

The 2002 dataset were processed directly in J2000 coordinates with an updated position for the phase calibration source $0955+697$ which has been measured to better than 1 mas [1]. In order to astrometrically align the 1992 data, they were first precessed to J2000 and corrections made to the nominal pointing center in order to account for the error in the original assumed position for $0955+697$ (several tens of mas). Finally, the peak of the most compact source in M82 (41.95+575) as measured in the 1992 image was aligned under the peak as seen in the deep 2002 image; involving a positional translation of less than 2 mas. After alignment on $41.95+575$, the peaks 


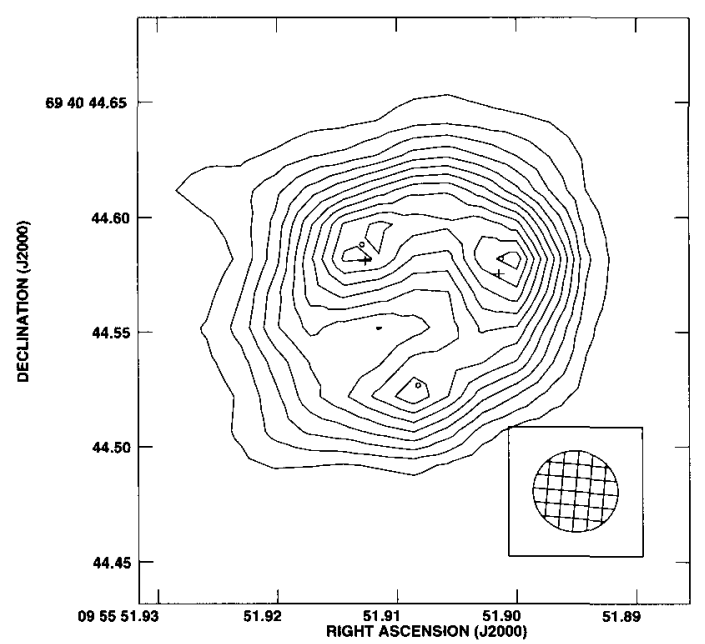

Fig. 2. Map of SNR $43.18+585$ from the deep 2002 epoch MERLIN dataset convolved with a 35 mas circular beam, with contours at $(1,2,3, \ldots .12) \times 50 \mu \mathrm{Jy}_{\text {beam }}{ }^{-1}$. Fitted positions for the 2002 epoch are shown as circles. Fitted positions for the 1992 epoch are shown as crosses.

of emission for the next two most compact SNR in M82 (43.31+592 and $44.01+596$ ) were found to align between the two epochs to better than 1 mas. Since SNR 44.01+596 lies 16.5 arcseconds from $41.95+575$ this implies than any relative stretch errors between the two epochs are at levels of $0.001 \%$ or better.

\section{Structural Evolution Between 1992 and 2002 for Compact SNR in M82}

In this section we summarize the structural evolution found between the images taken in epochs 1992.504 and 2002.249. The two most compact sources $(41.95+575$ and $43.31+592)$ have been studied for a number of years at mas angular resolution with Global VLBI. For these sources MERLIN provides an independent measure of the expansion velocities.

$41.95+575$ is the only source in M82 to show long-term systematic flux density variation with time and has been found to monotonically decrease in flux density at a rate of $8.5 \%$ year $^{-1}$ over many years. Between 1992 and 2002 the source was found to have decreased in flux density from $\sim 40 \mathrm{mJy}$ to $\sim 17 \mathrm{mJy}$ and the major axis of the source to have increased in size by 3.6 mas from $21.12 \pm 0.05$ to $24.71 \pm 0.07$ mas implying an expansion velocity of $2800 \pm 300 \mathrm{~km} \mathrm{~s}^{-1}$. This is in good agreement with global VLBI results and if the source is in free expansion, suggests a birth date around 1945. 


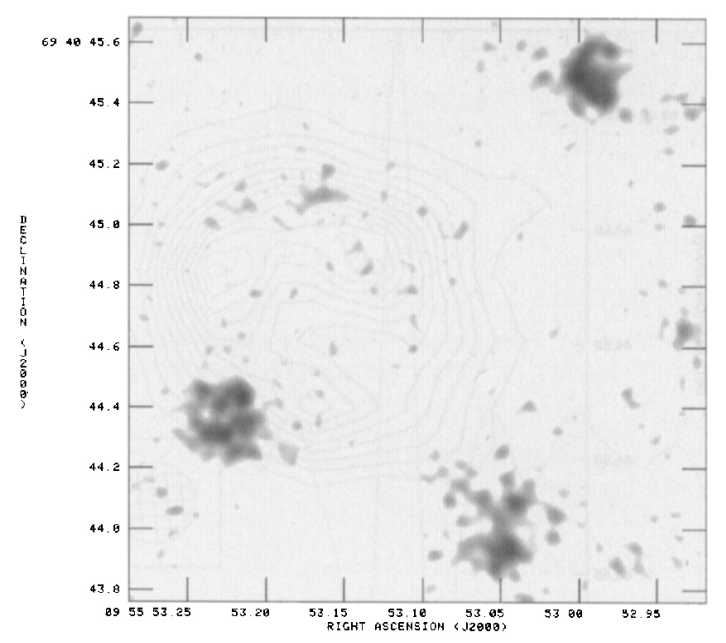

Fig. 3. SNR shell and partial shell structures in M82 revealed for the first time by the deep 2002 MERLIN image.

Between 1992 and 2002 this SNR 43.31+592 was found to have grown in size. The formal fit to the major axis is for an increase from $39.03 \pm 0.04$ to $49.52 \pm 0.02$ mas and for the minor axis an increase from $28.69 \pm 0.04$ to $40.43 \pm 0.02$ mas. Taking an average value, this implies an expansion speed of $8750 \pm 400 \mathrm{~km} \mathrm{~s}^{-1}$, again in good agreement with global VLBI results (J.D. Riley et al., these proceedings). There is also some evidence to suggest that the flux density of this SNR may have increased from $\sim 8.5$ to $\sim 10.2 \mathrm{mJy}$ between the two epochs. If the SNR is in free expansion this high velocity makes it the youngest SNR in M82 with a birth date around 1963.

SNR $43.18+585$ is substantially resolved by MERLIN and positional fits to the peaks of emission as seen in the 1992 and 2002 epochs (See Fig. 2) together with integrated annular profiles, have shown that these peaks have moved outward by $7.05 \pm 0.15$ mas in the 9.75 years between the epochs of the images implying an expansion velocity of $10,500 \pm 750 \mathrm{~km} \mathrm{~s}^{-1}$. If the SNR is in free expansion, with a measured mean radius of 37.7 mas in 2002, the source has a birth date around 1949. This is the first time that a reliable expansion velocity has been found for this SNR.

SNR $44.01+596$ has a somewhat disrupted shell-like radio structure. However, a comparison between the images from the two epochs shows that the major axis has increased by $3.40 \pm 0.05$ mas, and the minor axis by $2.70 \pm 0.05$ mas. This yields a relatively low expansion velocity of $2400 \pm 250 \mathrm{~km} \mathrm{~s}^{-1}$. Assuming free expansion gives an age in excess of 160 years. Again, this is the first time that an expansion velocity has been found for this SNR.

SNR $45.17+612$ shows only a partial shell structure. The peak of emission in the deep 2002 epoch image is found to be significantly displaced from that of the 1992 image by $\sim 9.5$ mas. This implies velocities $\sim 7500 \mathrm{~km} \mathrm{~s}^{-1}$. 
However, the direction of displacement does not appear to be radial (so far as this can be discerned from a partial shell structure). The interpretation of the measured motion is therefore unclear in this object.

The 2002 epoch image has revealed the detailed radio structures of many other SNR in M82 for the first time. However the poor signal-to-noise ratio of the original 1992 maps prevents reliable expansion measurements from being achieved. For these weaker SNR, later epoch deep images will be required before expansions velocities can be derived. These are planned, and with the advent of $e$-MERLIN, we expect to make such measurements for the majority of SNR in the central region of M82 over the next decade.

\section{Conclusions}

We have successfully measured the expansion velocities of the four most compact SNR in the central nuclear region of M82 over a 9.75 year period between 1992 and 2002 . The velocities found range from around $2500 \mathrm{kms}^{-1}$ to in excess of $10000 \mathrm{kms}^{-1}$ and do not appear to be related directly to the size or implied age of the remnant. We confirm the velocities found by VLBI for the two most compact remnants and report new results on two additional sources. The deep 2002 epoch MERLIN image will be used as the first epoch of a long-term study which should reveal the expansion velocities for the majority of established SNR in M82

\section{References}

1. A.J. Beasley: Private communication

2. A.R. McDonald et al. : Mon. Not. R. Astron. Soc. 322, 100 (2001)

3. T.W.B. Muxlow et al. : Mon. Not. R. Astron. Soc. 266, 455 (1994)

4. A. Pedlar et al. : Mon. Not. R. Astron. Soc. 307, 761 (1999)

5. J.D. Riley et al. : "Second epoch global VLBI observations of compact radio sources in the M82 starburst." In: These Proceedings. 